\title{
Ventricular assist devices for heart failure: a focus on patient selection and complications
}

This article was published in the following Dove Press journal:

Research Reports in Clinical Cardiology

9 September 2014

Number of times this article has been viewed

\author{
Manlio Cipriani \\ Vincenzo De Simone \\ Luciana D'Angelo \\ Enrico Perna \\ Marzia Lilliu \\ Virginia Bovolo \\ Fabrizio Oliva \\ Maria Frigerio \\ Cardiovascular and Thoracic \\ Department, A De Gasperis Niguarda \\ Ca' Granda Hospital, Milan, Italy
}

Correspondence: Manlio Cipriani

Cardiovascular and Thoracic

Department, A De Gasperis Niguarda

Ca' Granda Hospital, Milan, Italy

Email manlio.cipriani@ospedaleniguarda.it
Abstract: Heart transplantation represents the "gold standard" for the treatment of patients with end-stage heart failure, but remains challenged by inadequate donor supply, finite graft survival, and long-term complications arising from immunosuppressive therapy. In addition, a lot of patients waiting for a heart transplant experience clinical deterioration, and other patients become ineligible to undergo this treatment due to their age or relevant comorbidities. Left ventricular assist devices have emerged as a valid therapeutic option for advanced heart failure. In recent years, we have seen significant advances not only in the technologies available, but also in patient selection, indications for use, and management after implantation. Consequently, there has been an increase in the number of implants and an improvement in the survival rate and quality of life for these patients. At the same time, there are new challenges on the horizon. Patient selection is a difficult process, based on clinical and imaging parameters and risk scores, and more data are needed to refine patient selection criteria and the timing of the implant. Left ventricular assist device-related complications are still a serious problem, causing adverse events and hospital readmissions. Continuous progress in the development of these implantable devices, such as a further reduction in size and hopefully the abolition of the external driveline, will probably make ventricular assist devices an option also for less advanced stages of heart failure. Here, we discuss the current indications for left ventricular assist device implantation, patient selection criteria, and the most frequent complications associated with these devices.

Keywords: mechanical circulatory support, heart failure, destination therapy, bridge to transplantation, heart transplant

\section{Introduction}

End-stage heart failure (HF) refractory to medical therapy has reached epidemic proportions. Despite the improvement in medical treatment over the past half century, the prognosis remains poor, with an average 1-year mortality rate of 33\%. ${ }^{1}$ Heart transplantation represents the "gold standard" therapy for advanced and refractory HF with a 1-year survival rate of $81 \%,{ }^{2}$ but remains challenged by inadequate donor supply, finite graft survival, and the long-term complications of immunosuppressive therapy. In addition, the gap between the number of patients on waiting lists for heart transplants and the number of heart transplants per year is growing. Most of these patients experience clinical deterioration with end-organ dysfunction or are removed from the list because they have become ineligible for this treatment due to advanced age or severe comorbidities. Utilization of mechanical circulatory support (MCS) is currently a valid therapeutic option for end-stage HF. The evolution not only in technologies (ie, reduction in size, continuous flow), but also in patient selection and management 
after implantation, has improved patient outcomes. Currently, the survival rate 1 year after a left ventricular assist device (LVAD) implant is $80 \%$ and at 2 years is $70 \% .^{3}$ In addition, it is an immediately available therapy, compared with the heart transplant. If we also consider the growing experience with these devices, it is easy to understand the increasing use of this treatment (Figure 1). However, challenges continue with regard to patient selection criteria for implantation (not too late and not too early) and several LVAD-correlated complications (almost $70 \%$ of patients present an LVAD-related complication 1 year after surgery). ${ }^{3}$ This review describes the current state of LVAD devices, examining these points in particular.

\section{Patient selection: impact of guidelines and INTERMACS profiles}

Indications for MCS device implantation are described in Table 1. Several strategies are used to select appropriate candidates for LVAD referral. Because these patients usually have mortality similar to that of pretransplant patients, using guidelines similar to those for heart transplants is a reasonable option. ${ }^{4}$

According to the major guidelines and statements, the definition of patients eligible for MCS includes those with clinically significant circulatory compromise who require special care, including consideration for heart transplantation, continuous intravenous inotropic therapy, or admission to a hospice. Searching for specific indications between the last US and European guidelines, the former suggest MCS in stage D patients and refer substantially to a previously published statement for a more precise description of an eligible patient, while the latter define a more accurate indication on the basis of several parameters and values (Table 2). A common feature is to give priority to the bridge to transplant therapy strategy (respectively Class IIa and Class I) rather than the destination therapy strategy (in both cases Class IIa, level of evidence B). ${ }^{5,6}$

European Society of Cardiology guidelines recommend consideration of destination therapy in carefully selected patients who have end-stage HF despite optimal pharmacological and device therapy and who are not suitable for heart transplantation, but are expected to survive more than 1 year and have good functional status with LVAD therapy. The American College of Cardiology/American Heart Association have restrictive guidelines for LVAD placement as destination therapy, suggesting an expected 1-year survival rate of $50 \%$ despite medical therapies before consideration for device implantation. ${ }^{7}$
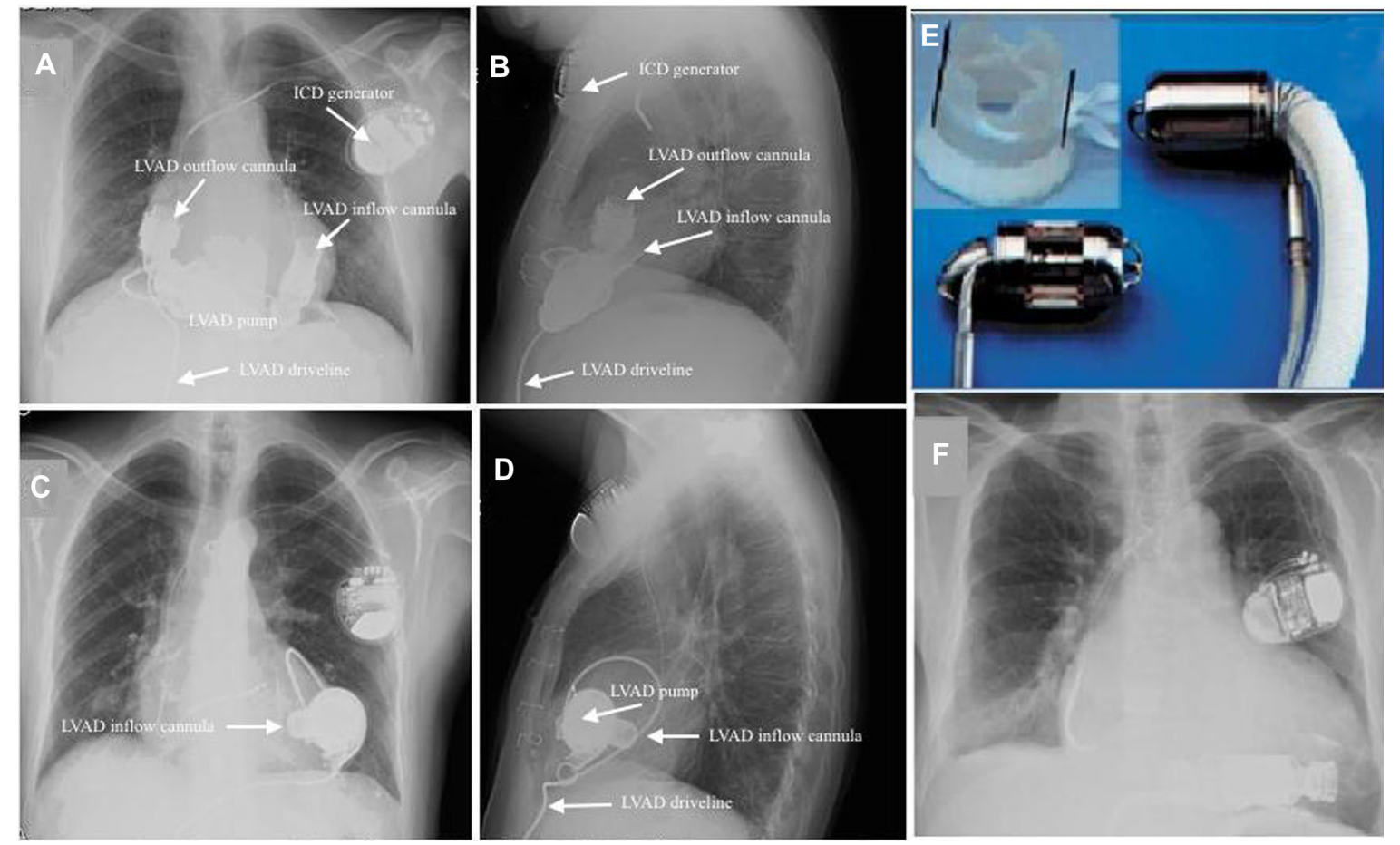

Figure I Postoperative chest X-ray illustrating a HeartMate II LVAD and an HVAD.

Notes: (A) Posterior-anterior LVAD; (B) lateral LVAD; (C) posterior anterior HVAD; (D) lateral HVAD. These are actually the two most frequently implanted devices in our center (Cardiovascular and Thoracic Department, A De Gasperis Niguarda Ca' Granda Hospital, Milan, Italy); (E) shows the Jarvik device; (F) shows a chest X-ray of an Incor LVAD.

Abbreviations: HVAD, heartware ventricular assist device; LVAD, left ventricular assist device; ICD, implantable cardiac defibrillator. 
Table I Indications for mechanical circulatory support

Bridge to Use of MCS in patients with drug-refractory acute decision circulatory collapse and at immediate risk of death to sustain life until a full clinical evaluation can be completed and additional therapeutic options can be evaluated.

Bridge to candidacy Bridge to transplant Bridge to recovery Destination therapy Use of MCS to improve end-organ function in order to make an ineligible patient eligible for transplantation. Use of MCS to keep a patient at high risk of death before transplantation alive until a donor organ becomes available. Use of MCS to keep patient alive until intrinsic cardiac function recovers sufficiently to remove MCS. Long-term use of MCS as an alternative to transplantation in patients with end-stage heart failure ineligible for transplantation.

Note: Data from Yancy et al. ${ }^{5}$

Abbreviation: MCS, mechanical circulatory support.

INTERMACS (the Interagency Registry for Mechanically Assisted Circulatory Support), which follows all long-term MCS systems in the USA, has defined patient profiles (Table 3 ) that can identify risks associated with the timing of the implant. They indicate that patients with cardiogenic shock (INTERMACS 1, or "crash and burn") may be too sick for permanent LVAD support. These patients have the highest risk of post-implantation mortality compared with patients presenting less severe HF (ie, INTERMACS patient profiles 2-7). The fifth annual report from INTERMACS showed that fewer emergency implantations are performed in hemodynamically unstable patients, confirming the recognition of the high mortality risk in this group. In this registry, the proportion of patients in progressive cardiac decompensation (level 2) or cardiogenic shock (level 1) at the time of implant has decreased from approximately $64 \%$ before 2011 to just under 54\% in 2012. Thus, for this population, consideration should be given to immediate stabilization with biventricular support, using temporary percutaneous or surgically placed systems or other appropriate treatments, to optimize their condition before permanent LVAD implant, if possible. Most patients who are stable on inotropes in INTERMACS category 3 would be appropriate candidates, and would likely benefit from LVAD therapy. ${ }^{3}$

On the other hand, profile 6-7 patients, who by definition have advanced New York Heart Association class III symptoms, are generally considered too well for MCS on the basis of current data. During the past year, the medical arm of INTERMACS (MEDAMACS) has been developed to assess medically treated patients who might become candidates for MCS devices. The intent is to investigate outcomes in these patients to better understand the "gray" area between medical treatment and ventricular assist device (VAD) therapy, particularly for INTERMACS levels 4-6. This registry was launched in January 2013, with 12 hospitals identified for the initial pilot study.

The INTERMACS classification scheme also includes modifiers for arrhythmia, frequent hospital admissions ("frequent flyers"), and temporary circulatory support, allowing increased consideration for patients affected by those factors that accelerate the risk of death (Table 4).

\section{Patient selection LVAD-responsive and LVAD-independent frailty}

Frailty is the aggregation of subclinical physiological insults across many organ systems, resulting in a syndrome of heightened vulnerability in the face of stress. ${ }^{8}$ Systolic and diastolic dysfunction contributes to LVAD-responsive frailty; this component can be treated by the device, unloading the left ventricle and reducing pulmonary capillary wedge pressure and right atrial pressure. However, many patients with advanced HF may be frail, due to illness related to extracardiac factors (aging, cancer, lung disease, diabetes, cirrhosis, peripheral vascular disease, neurological disease), which is not treatable with LVAD (LVAD-independent frailty). Therefore, a patient with primarily LVAD-responsive frailty is likely to have a good outcome if he or she survives the early postoperative period; in contrast, a patient with primarily LVAD-independent frailty is at greater risk of death, complications (eg, stroke, gastrointestinal bleed, or chronic hemolytic anemia), and/or persistently poor functional status after LVAD placement. ${ }^{9}$

\section{Risk scores for prediction of survival}

The development of a valid risk score to predict long-term survival after LVAD implantation has been a challenge in recent years (Table 5). The Destination Therapy Risk Score originally derives from a group of patients implanted with older generation devices, and considers a lot of variables, including laboratory values (international normalized ratio, creatinine/blood urea nitrogen, aspartate aminotransferase, albumin, platelet count, hematocrit), hemodynamic parameters (pulmonary artery pressure), and age. A recent study has concluded that it provides poor discrimination of mortality for bridge to transplant patients and only modest discrimination for destination therapy patients receiving continuous flow LVAD. ${ }^{10}$

The Model for End-Stage Liver Disease (MELD) score considers bilirubin, creatinine, and international normalized ratio. ${ }^{11}$ An alternative to this system is the MELD XI, based only on creatinine and bilirubin, which is used in patients 


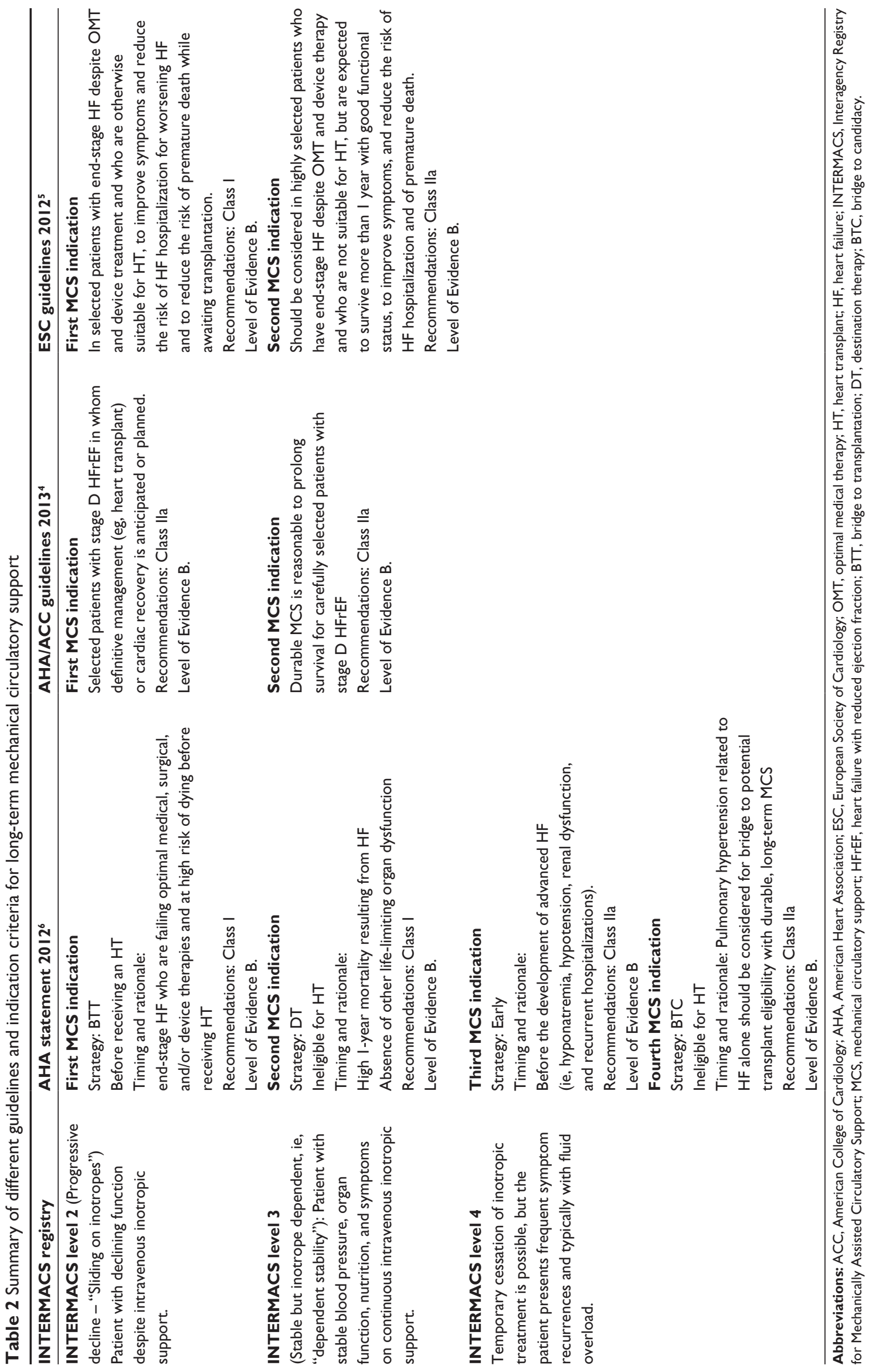


Table 3 INTERMACS patient profiles

\begin{tabular}{lll}
\hline Level & Description & Time to MCS \\
\hline I & "Crashing and burning" - critical cardiogenic shock & Within hours \\
2 & "Progressive decline" - inotrope dependence with continuing deterioration & Within a few days \\
3 & inotropes (patients stable on temporary circulatory support without inotropes are within this profile) \\
4 & "Recurrent advanced heart failure" - "recurrent" rather than "refractory" decompensation \\
5 & "Exertion intolerant" - describes patients who are comfortable at rest but are intolerant of exercise \\
7 & "Exertion limited" - a patient who is able to do some mild activity but fatigue results within a few & Wariable \\
& "Advanced NYHA 3" - describes patients who are clinically stable with a reasonable level of
\end{tabular}

Abbreviations: MCS, mechanical circulatory support; NYHA, New York Heart Association functional class; INTERMACS, Interagency Registry for Mechanically Assisted Circulatory Support.

on oral anticoagulant therapy. Patients with a MELD score below 17 have a survival advantage over those with a score of 17 or above, and MELD XI has proven to be a similar predictor of survival. The coefficients used for MELD score calculation were derived from patients with multifactorial liver disease and no documented cardiac dysfunction.

In contrast, the Heart Mate II Risk Score (HMRS) is derived from patients with advanced HF. ${ }^{12}$ The score components are age, international normalized ratio, serum albumin, creatinine, and implant center LVAD experience. It has a lot of advantages compared with the older scores. It is easy to calculate with simply obtained data. It is independent of device strategy (bridge to transplant or destination therapy). The variables (except for age) are parameters of organ function that can be optimized, so pharmacological strategies or mechanical support can be used to obtain better renal function (serum creatinine), better hepatic/right ventricular function (international normalized ratio), and a better inflammatory/nutritional state (albumin). The HMRS score cutoffs distinguish three risk groups: a lowrisk group (HRMS $<1.58$ ) with a 90 -day mortality of $4 \%$; a medium-risk group $(1.58 \leq$ HMRS $\leq 2.48)$ with a mortality of $16 \%$; and a high-risk group (HMRS $>2.48$ ) with a mortality of $29 \%$. Compared with INTERMACS profiles, the results of this score seem to demonstrate a stronger predictive impact of preserved end-organ function (however achieved) more than the use of preoperative vasopressors, inotropes, an intra-aortic balloon pump, or a ventilator. ${ }^{13}$

The CRITT (C, central venous pressure; $\mathrm{R}$, right ventricular dysfunction; I, preoperative mechanical ventilation/ intubation; $\mathrm{T}$, severe tricuspid regurgitation; $\mathrm{T}$, tachycardia) score is another score that can be quickly calculated at the bedside, by considering central venous pressure greater than $15 \mathrm{mmHg}$, severe right ventricular dysfunction, preoperative mechanical ventilation, severe tricuspid regurgitation, and tachycardia. A score less than 2 is highly predictive of the ability to tolerate an isolated LVAD. ${ }^{14}$

In conclusion, while we fully acknowledge the importance of our clinical judgment, risk scores represent an additional tool to predict survival after LVAD implantation and to identify targets for therapy before surgery.

\section{Right ventricular failure}

Right ventricular failure (RVF) following LVAD therapy is a strong predictor of mortality and is associated with a greater risk of bleeding, a higher rate of renal insufficiency, and longer hospitalizations; consequently, identification of patients at high risk for RVF is essential. ${ }^{15}$ Recently, the concepts of the "vulnerable" right ventricle and of the "frail" patient

Table 4 Modifiers for INTERMACS profiles

\begin{tabular}{lll}
\hline Modifier & Description & INTERMACS profiles modified \\
\hline TCS & $\begin{array}{l}\text { Temporary circulatory support can modify only patients in hospital; } \\
\text { includes IABP, ECMO, TandemHeart, Levitronix, Impella. }\end{array}$ & Possible profiles to modify I, 2, 3 in hospital. \\
A & Arrhythmia can modify any profile. Recurrent ventricular & Any profile. \\
& tachyarrhythmias that contributed to clinical compromise also with & 3 if at home, 4, 5, 6. \\
FF & ICD shock or requirement for external defibrillator are included. & A frequent flyer would rarely be profile 7. \\
& temergency visits or hospitalizations for diuretics, ultrafiltration, or & \\
\hline
\end{tabular}

Abbreviations: ICD, implantable cardioverter-defibrillator; TCS, temporary circulatory support; A, arrhythmia; FF, frequent flyer; IABP, intra-aortic balloon pump; ECMO, extracorporeal membrane oxygenation; INTERMACS, Interagency Registry for Mechanically Assisted Circulatory Support. 
Table 5 Risk scores predicting survival after LVAD implantation

\begin{tabular}{|c|c|c|}
\hline Risk score & Variables & Outcomes predicted \\
\hline HeartMate II risk & - Older age & Independent predictors of 90 days \\
\hline \multirow[t]{4}{*}{ score $^{12}$} & - Lower albumin & mortality. \\
\hline & - Higher creatinine & Independent predictors of 2 years mortality (only \\
\hline & - Higher INR & older age and center experience) \\
\hline & - Less center experience & \\
\hline \multirow[t]{9}{*}{ DT risk score ${ }^{10}$} & - Platelet count $\leq 148 \times 103 / \mu \mathrm{L}$ & 90 days in-hospital mortality \\
\hline & - Albumin $\leq 3.3 \mathrm{~g} / \mathrm{dL}$ & \\
\hline & $\cdot$ INR $>$ I.I & \\
\hline & - Vasodilator therapy at time of implantation & \\
\hline & - Mean PAP $\leq 25 \mathrm{mmHg}$ & \\
\hline & - $\mathrm{AST}>45 \mathrm{U} / \mathrm{L}$ & \\
\hline & - Hematocrit $\leq 34 \%$ & \\
\hline & - $\mathrm{BUN}>5 \mathrm{I} \mathrm{U} / \mathrm{dL}$ & \\
\hline & - No intravenous inotropes & \\
\hline \multirow[t]{5}{*}{ MELD risk score" } & - Bilirubin & Operative mortality $(60 \%$ increase for \\
\hline & - INR & every 5 -unit increase in score) \\
\hline & - Creatinine & 6-months mortality (patients with a preoperative \\
\hline & & MELD $\geq 17$ had increase in 6-months mortality \\
\hline & & compared with patients whose MELD was <17) \\
\hline \multirow[t]{2}{*}{ MELD-XI risk score" } & - Bilirubin & 6-months mortality (patients with a preoperative \\
\hline & - Creatinine & $\begin{array}{l}\text { MELD-XI } \geq 17 \text { had increase in } 6 \text {-months mortality } \\
\text { compared with patients whose MELD- } X I \text { was }<17)\end{array}$ \\
\hline Right ventricular failure & - CVP/PAP ratio $>0.63$ & Independent predictors of right ventricular failure \\
\hline \multirow[t]{2}{*}{ risk model ${ }^{23}$} & - Preoperative ventilator support & following LVAD implantation \\
\hline & - $\mathrm{BUN}>39 \mathrm{mg} / \mathrm{dL}$ & \\
\hline \multirow[t]{5}{*}{ CRITT score $\mathrm{e}^{14}$} & - CVP greater than 15 mmHg (C) & A score less than 2 is highly predictive \\
\hline & - Severe RV dysfunction (R) & of the ability to tolerate isolated \\
\hline & - Preoperative intubation (I) & LVAD therapy \\
\hline & - Severe tricuspid regurgitation $(\mathrm{T})$ & \\
\hline & - Tachycardia (T) & \\
\hline
\end{tabular}

Abbreviations: LVAD, left ventricular assist device; INR, international normalized ratio; PAP, pulmonary artery pressure; AST, aspartate transaminase; BUN, blood urea nitrogen; CVP, central venous pressure; DT, destination therapy; VAD, ventricular assist device; BTT, bridge to transplantation; RV, right ventricle; MELD, Model for End Stage Liver Disease; MELD-XI, MELD excluding INR; C, central venous pressure; R, right ventricular dysfunction; I, preoperative mechanical ventilation/intubation; T, severe tricuspid regurgitation; $\mathrm{T}$, tachycardia.

Table 6 Multiparametric evaluation in patient selection for LVAD implant

\begin{tabular}{ll}
\hline RV vulnerability & Patient frailty \\
\hline Cardiac catheterization & Biochemical parameters \\
Low RVSWI $\left(<300 \mathrm{mmHg} / \mathrm{mL} / \mathrm{m}^{2}\right)$ & Bilirubin $\geq 2 \mathrm{mg} / \mathrm{dL}$ \\
High CVP with low PAP & Transaminase: AST $>45 \mathrm{mg} / \mathrm{dL}$ \\
CVP/PCWP $>0.63$ & $\begin{array}{l}\text { Albumin }<3.3 \mathrm{~g} / \mathrm{dL} \\
\text { Low total cholesterol } \\
\text { Renal function: }(\mathrm{BUN}>5 \mathrm{I} \mathrm{mg/dL} \\
\text { or creatinine }>2.3 \mathrm{mg} / \mathrm{dL})\end{array}$ \\
Clinical status \\
Low RV strain & Cachexia \\
Altered RV geometry & INTERMACS level I \\
Very low FAC RV ( $<35 \%)$ & \\
Moderate-severe tricuspid & \\
regurgitation & \\
Low contractile reserve \\
of right ventricle \\
(echo-dobutamine)
\end{tabular}

Abbreviations: AST, aspartate aminotransferase; BUN, blood urea nitrogen; LVAD, left ventricular assist device; RV, right ventricle; RVSWI, right ventricle stroke work index; CVP, central venous pressure; PAP, pulmonary artery pressure; PCWP, pulmonary capillary wedge pressure; FAC, fractional area change; INTERMACS, Interagency Registry for Mechanically Assisted Circulatory Support. have been introduced (Table 6); not only is a complete study of right ventricular function using parameters derived from echocardiography and right heart catheterization necessary, but also evaluation of the clinical status of the patient and the presence of organ dysfunction. ${ }^{16}$

Several studies have also considered the impact of the indication for LVAD (destination therapy seems to be a risk factor compared with bridge to transplant) and the type of cardiomyopathy (the risk of right ventricular failure after LVAD occurs, especially in patients with nonischemic causes, where both ventricles are often equally impaired). ${ }^{17,18}$

Echocardiography remains the primary imaging modality for evaluation of the right ventricle (Table 7), but research of less load-dependent parameters is a challenge. In this regard, more recently, the study by Grant et al shows the role of right ventricular strain; it represents a measure of deformation of the ventricular wall normalized to its original shape, and is more strongly correlated with postoperative RVF than traditional echocardiographic parameters. ${ }^{19}$ 
Table 7 Echocardiographic reference limits for recommended measures of right heart chamber

\begin{tabular}{ll}
\hline Measure & Abnormal values \\
\hline Basal RV diameter & $>4.2 \mathrm{~cm}$ \\
RV subcostal wall thickness & $>0.5 \mathrm{~cm}$ \\
RVOT in PSAX distal diameter & $>2.7 \mathrm{~cm}$ \\
RVOT in PLAX proximal diameter & $>3.3 \mathrm{~cm}$ \\
TAPSE & $<1.6 \mathrm{~cm}$ \\
Pulsed Doppler peak velocity at the annulus & $<10 \mathrm{~cm} / \mathrm{sec}$ \\
FAC (\%) & $<35 \%$ \\
Tei index & $<0.44$ pulsed Doppler \\
& $<0.55 \mathrm{TDI}$ \\
Longitudinal 2D strain & $<20 \%$ \\
EF-3D & $<44 \%$ \\
\hline
\end{tabular}

Abbreviations: RV, right ventricle; RVOT, right ventricular outflow tract; PSAX, parasternal short axis; PLAX, parasternal long axis; FAC, fractional area change; TAPSE, tricuspid annular plane systolic excursion; TDI, tissue Doppler index; EF, ejection fraction; 2D, two-dimensional; 3D, three-dimensional.

Attempting to predict the impact of unloading following LVAD implantation on the right ventricle, Dandel et al introduced a load adaptation index, a parameter considering the ratio between the end diastolic area of the right ventricle and long-axis length (AED/LED) and the tricuspid velocity-time integral (VTITR). ${ }^{20}$

Another study has shown that preoperative right ventricular fractional area change, estimated right atrial pressure, and left atrial volume may be combined into a simple echocardiographic scoring system to provide an additional tool for risk-stratifying patients when evaluating for LVAD implantation. ${ }^{21}$

We also report the experience of Deswarte et al with a low-dose dobutamine stress echocardiogram, in which an increase in tricuspid annular plane systolic excursion score by $40 \%$ and/or an increase in pulmonary artery systolic pressure over $30 \%$ ruled out post-LVAD right ventricular failure with $100 \%$ specificity and sensitivity. ${ }^{22}$

Tricuspid regurgitation is also correlated with postoperative RVF, and is a clear consequence of right ventricular dilatation. A lot of studies show a more aggressive treatment of tricuspid regurgitation (from moderate to severe) with annuloplasty; this strategy may reduce volume loading of the right ventricle and improve right ventricular contractility. ${ }^{23}$

In conclusion, many parameters derived from echocardiography have been described, but limited data have been obtained due to the retrospective/single-center nature of these studies.

With regard to hemodynamic parameters (Table 8), central venous pressure $>15 \mathrm{mmHg}$, a right ventricular stroke work index $<300 \mathrm{mmHg} / \mathrm{mL} / \mathrm{m}^{2}$, and a central venous pressure/pulmonary capillary wedge pressure ratio $>0.63$ have
Table 8 Normal values at right heart catheterization

\begin{tabular}{ll}
\hline \multicolumn{1}{c}{ Normal values } \\
\hline RAP & $0-7 \mathrm{mmHg}$ \\
MAP & $10-20 \mathrm{mmHg}$ \\
PCWP & $6-15 \mathrm{mmHg}$ \\
Cl & $2.5-3.6 \mathrm{~L} / \mathrm{min} / \mathrm{m}^{2}$ \\
Right ventricular function \\
RAP/PCWP & $>0.63$ (right ventricular failure $)^{22}$ \\
SVI & $\mathrm{SVI}=\mathrm{Cl} /$ heart rate $\times \mathrm{I}, 000=33-47 \mathrm{~mL} / \mathrm{m}^{2} /$ beat \\
RVSWI & RVSWI $=(\mathrm{MAP}-\mathrm{RAP}) \times \mathrm{SVI} \times 0.0136=4-12 \mathrm{~g} / \mathrm{m} / \mathrm{beat} / \mathrm{m}^{2}$ \\
& RVSWI $=(\mathrm{MAP}-\mathrm{RAP}) \times \mathrm{SVI}=300-900 \mathrm{mmHg} \times \mathrm{mL} / \mathrm{m}^{2}$ \\
& $<300 \mathrm{mmHg} \times \mathrm{mL} / \mathrm{m}^{2}(\text { right ventricular failure })^{22}$ \\
\hline
\end{tabular}

Abbreviations: RAP, right atrial pressure; MAP, pulmonary artery mean pressure; PCWP, pulmonary capillary wedge pressure; $\mathrm{Cl}$, cardiac index; SVI, stroke volume index; RVSWI, right ventricular stroke work index.

been identified as predictors of a higher risk of RVF. ${ }^{24}$ These parameters are strongly influenced by the loading conditions of the right ventricle (preload = right atrial pressure; afterload $=$ mean pulmonary pressure and transpulmonary gradient). For this reason, before evaluating the hemodynamic profile, it is essential to optimize the state of the patient's compensation and management of volume overload (using intravenous diuretics and inotropes or vasodilators).

Patients with evidence of multiorgan dysfunction at the time of LVAD implantation are at higher risk of RVF. In particular, those who require preoperative ventilator support, intravenous vasopressors or an intra-aortic balloon pump for circulation support, or have evidence of liver (high bilirubin or aspartate aminotransferase levels) or renal (elevated serum creatinine) impairment, appear to be particularly vulnerable to postoperative RVF. ${ }^{17,19,25}$ Also, patients with coagulation abnormalities and/or hypoalbuminemia may be at highest risk of developing postoperative RVF. ${ }^{17,25}$

With regard to the clinical status of the patient, right ventricular dysfunction and cardiac cachexia often coexist and have an additional adverse impact. Fat loss is clearly a predictor of an adverse outcome, but it is unclear whether it is a surrogate of enhanced catabolism or if adipose tissue is cardioprotective in an HF context. ${ }^{26}$

In conclusion, the prediction of RVF post-LVAD implant is based on the contemporary evaluation of echocardiographic, hemodynamic, biochemical, and clinical parameters, but a universal risk score does not yet exist. The International Society for Heart and Lung Transplantation Guidelines for Mechanical Circulatory Support of 2013 recommend that patients with evidence of right ventricular dysfunction preoperatively should be considered for aggressive management, which may include diuretics, ultrafiltration, inotropes, an intra-aortic balloon pump, or other short-term mechanical support. Once optimized, right ventricular function should be reassessed. ${ }^{27}$ 


\section{LVAD-related complications Bleeding}

Bleeding is one of the most common complications after LVAD implantation. In the INTERMACS registry, the rate of bleeding requiring red blood cell transfusion post-US Food and Drug Administration approval was 0.84, and the prevalence is established at around 1.14 major bleeding events per patient-year. ${ }^{28}$

Due to the large surface area of artificial material that the blood contacts, anticoagulation and antiplatelet therapies are necessary to prevent thrombus formation. The use of these drugs cannot entirely explain bleeding in LVAD patients. The evidence shows that acquired von Willebrand syndrome can play a considerable role in the genesis of hemorrhage and can explain the different bleeding rates observed in the two groups of LVAD recipients..$^{29,30}$ It has been demonstrated that, in continuous flow device carriers, the level of large von Willebrand multimer drops, perhaps due to narrow pulse pressure. The LVAD impeller mechanism provokes the demolition of large multimers, leading to acquired von Willebrand disease. Bleeding is also explained by the presence of reduced collagen binding capacity and low ristocetin cofactor activity (which measures the binding of the von Willebrand factor to platelets) and by the effect of antiplatelet and anticoagulation drugs. These coagulation disorders persist even 10 weeks after LVAD implantation and even after heart transplant in bridge to transplant patients.

The most common site of bleeding is the gastrointestinal tract ( $45 \%$ of bleeding requiring transfusion according to recent data), followed by the wound site $(12 \%)$ and the nasal mucosae (4\%). Although less common, the most feared source of bleeding is undoubtedly the intracranial site, and the overall prevalence of hemorrhagic stroke reaches $8 \%$. In multivariate analyses, Boyle et al identified a few independent risk factors for bleeding, ie, age $>65$ years, female sex, ischemic etiology, and hematocrit $\leq 31 \% .^{31}$

In a retrospective analysis, gastrointestinal bleeding originated in $57 \%$ of cases from the upper tract (gastric erosion is responsible for $54 \%$ of bleeding, followed by arteriovenous malformations and gastric ulcers) and in 35\% of cases from the lower tract (originating equally from small bowel angiodysplasias and cecal or rectal ulcers). ${ }^{32}$ For the management of bleeding, adapting LVAD parameters can be an overriding factor: usually, anticoagulation is temporarily discontinued and pump flow speed is reduced (in order to increase pulse pressure). Using this strategy, John et al did not encounter any recurrence of gastrointestinal bleeding nor of thromboembolism. ${ }^{33}$ Anticoagulation reversal using vitamin $\mathrm{K}$ or plasma seems to be safe. ${ }^{34}$

\section{Hemolysis}

Hemolysis is diagnosed with a plasma-free hemoglobin value $\geq 40 \mathrm{mg} / \mathrm{dL}$ in association with clinical signs and symptoms (such as anemia, low hematocrit, hyperbilirubinemia). ${ }^{35}$ Recently, it has been demonstrated that monitoring lactate dehydrogenase values (cutoff $\geq 600 \mathrm{UI} / \mathrm{L}$ ) permits earlier identification of adverse events. Clinical signs of hemolysis are often the early indicators of pump thrombus, particularly if associated with increased pump power utilization. ${ }^{36}$ Hemolysis, acting as a marker of pump thrombosis, is associated with decreased survival $(38.9 \%$ at 1 year $){ }^{37}$

\section{Thrombosis}

Pump thrombosis is an uncommon but potentially catastrophic event, as a cause of device exchange or death. According to the INTERMACS registry, freedom from definite or probable pump thrombosis is $95 \%$ at 1 year and $92 \%$ at 2 years; the hazard function reveals an early hazard peaking at 1 to 2 months and a gradually increasing late hazard..$^{38}$ Pump thrombosis is a multifactorial phenomenon, including mechanically induced thrombosis (due to a severely abnormal inflow cannula position or bend relief disconnect with deformed outflow graft or hypercoagulable disorder) and "nonmechanical" device thrombosis (without clear proximate etiology, sometimes occurring after infections). ${ }^{39}$ The identified risk factors for pump thrombosis are: later implant year, marked elevation of lactate dehydrogenase at 1 month, younger age, worse renal function, a larger patient, and less severe ventricular dysfunction. The survival rate after pump replacement for thrombosis was $56 \%$ at 2 years compared with $69 \%$ after the primary implant. Freedom from infection and cerebrovascular accident were significantly worse after pump exchange compared with primary implant. No difference was detected in freedom from gastrointestinal bleeding (data from INTERMACS registry). The diagnosis of pump thrombosis, aside from hemolysis, relies on echocardiography and a cardiovascular computed tomography scan. Echocardiography can detect a reduction in cannula diastolic flow velocity and an increased systolicdiastolic flow velocity ratio; further, a ramp test has been developed to clarify suspicion of thrombosis. ${ }^{40}$ This protocol measures left and right ventricular decompression and valve function during pump speed variations; minimal changes in left ventricular end-diastolic diameter with increasing speed and aortic valve closing at higher speed were predictors of pump thrombosis. No standard algorithm exists for diagnosis 
of thrombosis with a computed tomography scan, but it can help in identifying cannula malpositioning and outflow graft thrombosis. ${ }^{41}$ If pump thrombosis is suspected, urgent surgical pump replacement should be considered. Thrombolytic intraventricular therapy has also been used with success, but should be carefully evaluated because of the risk of hemorrhagic and thromboembolic complications. ${ }^{42}$

\section{Right heart failure}

Right heart failure (RHF) is probably the most feared complication, and its incidence is about $18 \%$ in post-US Food and Drug Administration approval data. ${ }^{24}$ It is defined as the presence of symptoms and signs of persistent right ventricular dysfunction (central venous pressure $>18 \mathrm{mmHg}$ with a cardiac index $<2.3 \mathrm{~L} / \mathrm{min} / \mathrm{m}^{2}$ in the absence of elevated left atrial/pulmonary capillary wedge pressure, tamponade, ventricular arrhythmias or pneumothorax) requiring right ventricular assist device (RVAD) implantation, inhaled nitric oxide, or inotropic therapy for a duration of more than 1 week at any time after LVAD implantation. ${ }^{35}$

A severity scale for RHF grading has also been developed: ${ }^{43,44}$

- severe, requiring RVAD implantation

- moderate, warranting inotropes or use of intravenous or inhaled pulmonary vasodilator (inhaled nitric oxide or prostaglandin E)

- mild, indicated by two of the following criteria: central venous pressure $>18 \mathrm{mmHg}$ or mean right atrial pressure $>18 \mathrm{mmHg}$; cardiac index $<2.3 \mathrm{~L} / \mathrm{min} / \mathrm{m}^{2}$; ascites or evidence of moderate to worse peripheral edema; evidence of elevated central venous pressure by echocardiography (dilated inferior vena cava without collapse) and upon physical examination (signs of increased jugular venous pressure).

RHF after LVAD implantation can also be classified as intraoperative, early, or late ( $>14$ days after surgery) according to the time of onset.

It has been demonstrated that acute unloading of the left ventricle leads to a septal shift that alters right ventricular shape and size, thereby affecting its contractility. ${ }^{45}$ In addition, latent right ventricular dysfunction can be unmasked with the increase in right-sided preload afforded by LVAD perfusion. RHF increases the mortality and morbidity of patients, leading to end-organ dysfunction, with a $20 \%$ reduction in 1-year survival for individuals who developed early RHF compared with those who did not. If severe RHF occurs, right ventricular mechanical support is the only effective therapy for refractory RHF; nevertheless, biventricular assist device support is burdened by poor outcomes, due to the high rate of infections and embolic events. Saito et al recently demonstrated that a temporary right VAD allows us to identify patients who could be weaned from right VAD support: about $42 \%$ of patients requiring right ventricular mechanical support were successfully weaned, with a survival rate comparable with that for isolated LVAD support. ${ }^{46}$

\section{Aortic regurgitation}

One important adverse event is the development of aortic valve pathology during LVAD support. When an LVAD unloads the left ventricle, the opening of the aortic valve is diminished or abolished; the systemic pressure becomes minimally pulsatile, with a mean aortic pressure that is much higher than normal aortic diastolic pressure. This means that the aortic valve is exposed to increased transvalvular pressures (the difference between the left ventricular and aortic pressures). Normally, the aortic valve is accustomed to tolerating transvalvular pressure of $80 \mathrm{mmHg}$, but with continuous flow LVAD, this value increases by $25 \%$. This stress on the aortic valve may lead to some degree of degeneration, deterioration, and remodeling, with regurgitation and/ or fusion of the leaflets. ${ }^{47,48}$ About $80 \%$ of patients without preoperative valve regurgitation showed rapid development of mild to moderate aortic regurgitation following their LVAD implantation. ${ }^{49}$ This "de novo" aortic regurgitation induced by the LVAD is considered to have deleterious effects on pump efficiency and systemic output. In fact, this creates a circulatory loop of flow to the ventricle instead of the systemic circulation, diminishing the efficiency of support and the ventricular unloading..$^{50}$ Obviously, an increase in pump speed may initially compensate for the loss of systemic circulation. Once present in patients with a permanently closed aortic valve, aortic regurgitation manifests as a continuous flow toward the left ventricle during diastole as well as systole. Otherwise, if the aortic valve is allowed to open, aortic regurgitation manifests during diastole. Remarkably, in a small number of patients, the aortic regurgitation manifests only in the systolic phase of the cardiac cycle and probably would disappear at the opening of the aortic valve. ${ }^{49,51}$ The mechanism involved in this LVAD-related systolic aortic regurgitation is still unknown, but may involve a different mechanism associated with dynamic modification of the aortic annulus or valve as a result of turbulence in the ascending aorta. However, when aortic regurgitation becomes more severe, valve repair may eventually be inevitable. ${ }^{52}$ The next generation of LVAD will partially resolve this adverse event. In the HeartMate III the rotor speed will periodically depart 
from the value that has been initially set by the clinician in order to contribute a flow disruption that can mimic native cardiac contractility (30 "artificial beats" per minute, asynchronously with the heart). In this way, it will be possible to reduce the high levels of mean aortic pressure and stress on the valvular leaflets. ${ }^{53}$

\section{Arrhythmias}

LVAD patients are at risk for morbidity and mortality from atrial and ventricular arrhythmias. First of all, it is important to understand that an LVAD only assists the left ventricle. The right ventricular performance requirements remain unchanged. Although the LVAD will still be functioning during atrial and ventricular arrhythmias, right ventricular function might deteriorate significantly, leading to inadequate left ventricular filling and decompensated HF, syncope, or death. $^{54}$

\section{Atrial arrhythmias}

Patients with LVAD are not dependent on their atrial kick to fill the LVAD; the device creates a vacuum that draws blood into the pump throughout the cardiac cycle. However, patients may still decompensate with the loss of the right atrial kick, especially in the setting of poor right ventricular function. Because one of the risk factors for atrial fibrillation is left atrial distention from congestion and/or mitral regurgitation, decompression of the left ventricle and consequently of the left atrium by an LVAD should reduce the long-term risk of atrial fibrillation. Nevertheless, as atrial fibrillation increases the activity of the LVAD, the risk of atrial fibrillation remains high.

Treatment of atrial arrhythmias following MCS involves rate control (particularly with beta-blockers) or rhythm control in selected patients (especially with electrical cardioversion or with amiodarone). All patients with LVAD are already on warfarin to prevent pump thrombosis and thromboembolism, in the absence of contraindications to anticoagulation, and do not need adjustment of therapy. ${ }^{54,55}$

\section{Ventricular arrhythmias}

Ventricular arrhythmias affect at least one third of patients treated with long-term VAD support for advanced HF. ${ }^{56}$ Several factors affect the likelihood of ventricular arrhythmias occurring during VAD support. Of these, the most consistently observed risk factor is a history of ventricular arrhythmias prior to initiation of VAD support. Other risk factors for ventricular arrhythmias during VAD support include early increases in the QT interval, electrolyte disturbances, the absence of beta-blocker treatment, and a history of atrial fibrillation prior to initiation of VAD support. ${ }^{57}$

Three mechanisms cause these ventricular arrhythmias. The first is the effect due to the inflow cannula. With a continuous flow device, transient changes in venous return can result in negative pressure at the inflow cannula, leading to a suction event. Suction events can affect the interventricular septum or left ventricular free wall. This can cause mechanical stimulation of arrhythmia, tissue injury, and low flow. ${ }^{57,58}$ The specific circumstances in which suction is likely to occur include overdiuresis, bleeding, and overpumping; reduction of LVAD speed can be useful to reduce the likelihood of collapse of the septal wall. ${ }^{54}$ Secondly, myocardial fibrosis (antecedent scarring or localized injury induced by cannula placement) increases the propensity for arrhythmias. ${ }^{59}$ Use of angiotensin-converting enzyme inhibitors may reduce levels of fibrosis and collagen deposition. The third aspect is the electrophysiological adaptation to LVAD support. In fact, lots of studies have demonstrated how the ventricular unloading induced by LVAD can modify depolarization and repolarization of the left ventricle. In particular, the QRS complex tends to decrease, especially in the first week up to 6 months, and the QT interval is often prolonged. Betablockers and other antiarrhythmic drugs are frequently used. 57,60

In urgent cases, antiarrhythmic drugs are commonly used following the Advanced Life Support algorithm; also, electrical cardioversion is commonly performed and generally safe. Cardiopulmonary resuscitation is not recommended by the manufacturers secondary to potential cannula dislodgment, unless the pump is not working and the patient has lost blood pressure.

Many patients already have an implantable defibrillator for primary or secondary prevention, upon implantation of a LVAD. The real benefit of implantable defibrillators in VAD-supported patients is not yet clear. Inappropriate defibrillations appear to be relatively uncommon $(6.5 \%)$, but in some cases, reprogramming or replacement of the implantable defibrillator due to interaction with the VAD may be necessary. ${ }^{54}$

\section{LVAD infections}

Data from the INTERMACS registry identified infection as the cause of $16 \%$ of deaths post-LVAD implantation, second only to HF. In the population awaiting heart transplantation while on LVAD support, late infection was the reason for declining an organ in about $30 \%$ of cases. Among patients who have died of infection during LVAD support while 
awaiting transplantation, 29\% had a driveline infection. Many factors identify patients at higher risk for LVAD infection, including older age, nutritional status, immunosuppression, oral hygiene, diabetes, renal failure, and obesity. ${ }^{61}$ In 2010 , a core of group experts formed an International Society for Heart and Lung Transplantation Infection Diseases Working Group to develop agreed criteria for definitions of infections in VAD patients. ${ }^{62}$ These definitions divided infections in patients with LVAD into three sections, ie, VADspecific infections, VAD-related infections, and non-VAD infections.

\section{VAD-specific infections}

VAD-specific infections are the principal group. They may arise due to the hardware itself or the body surfaces that contain them, and include infections of the pump, cannula, anastomoses, pocket, and the percutaneous driveline or tunnel. However, the most prevalent of these is infection of the driveline. This percutaneous channel acts as the port of entry for external pathogens, which can then migrate deeper through this tunnel into host tissues. If this process is not stopped, pathogens can even arrive at the pump itself, causing pocket infection, which may be extremely difficult to eradicate or even to control.

Definite diagnosis of percutaneous entry site infections requires a positive culture obtained from the skin or the tissue or fluid surrounding the external housing of the pump. Gram-positive organisms, commonly Staphylococcus aureus, cause most infections, but Enterococcus and other Staphylococcal species are also frequently isolated. The Gramnegative pathogen most frequently isolated is Pseudomonas aeruginosa. Infections caused by Candida are associated with the highest mortality rate. ${ }^{63}$ In the case of a driveline infection, both local treatment with cleaning and systemic antibiotic treatment is indicated, preferably driven by bacterial cultures. In particular, administration of antibiotics is indicated in the event of fever, leukocytosis, pain, or drainage around the driveline entry site or its internal pathway. Such infections usually require a long course of parenteral treatment for 6 weeks or longer, depending on the response, followed by oral antibiotics. However, prevention is the most important way to ward off infection. Antibiotic prophylaxis is important before implantation and also for 24-48 hours after the procedure. The protocol for administration consists of a combination of vancomycin (1 g), ciprofloxacin (400 mg), and fluconazole (400 mg) dosed within 30 minutes of skin incision and continued until 48 hours after sternal closure using vancomycin (1 g every 12 hours), ciprofloxacin (400 mg every 12 hours), and fluconazole (400 mg every 24 hours), with adequate renal adjustment. ${ }^{61,64}$ The second aspect is the selection of the right exit site location. It is important to consider body habitus and eventual body weight changes, to prevent opening of the peritoneal cavity during implantation, and to create a small and clean wound in the skin. The driveline must be sutured to the skin and kept immobile, using drain attachment devices to avoid trauma. The third and most important point is driveline management after the intervention. Daily antiseptic cures are fundamental, and patients and caregivers must be educated and instructed in how to medicate the wound. Moreover, they should be aware of the signs of infection, so that treatment can be instituted early. ${ }^{61,65}$

Exchange of the device is a high-risk procedure. Only intractable cases, particularly in the presence of LVADassociated endocarditis, require device explantation and in a context of destination therapy. If the patient is still eligible for heart transplant, we prefer to place him or her on a more urgent status on the waiting list.

\section{VAD-related infections}

VAD-related infections refer to those infections not directly involving the VAD itself but probably occurring as a result of VAD implantation. This group includes infective endocarditis, bloodstream infections, mediastinitis, and sternal wound infection. ${ }^{62}$ Computed tomography techniques can show the exact position and extent of inflammatory tissue, and this can be useful to understand the real relationship between the infection site and one portion of the VAD system.

\section{Non-VAD infections}

Non-VAD infections are essentially "independent" of or not directly related to the presence of the VAD. These infections (most frequently lower respiratory tract infection, cholecystitis, Clostridium difficile infection, and urinary tract infection) occur in a sick population of immunecompromised hosts with underlying comorbidities such as diabetes, prolonged hospitalization, multiple drug regimens, and renal impairment. ${ }^{62}$

\section{Conclusion}

Patient selection for MCS continues to evolve along with the technology. The selection criteria for MCS are not static, and frequent reassessment of candidacy is required after changes in the patient's condition. Confirmation of adequate psychosocial support and the capacity for self-care is also crucial. Without this, successful surgery could be rendered futile in the long run. However, more data are needed to define 
better patient selection criteria and calculate the timing of the implant. Complications that accompany LVAD therapy, such as failure of the VAD secondary to the thrombosis device and infection of the external driveline, cannot be ignored. Continuous progress in the development of this implantable device, such as a further reduction in size and hopefully abolition of the external driveline, will probably make the VAD an option also for less advanced stages of HF.

\section{Disclosure}

The authors report no conflicts of interest in this work.

\section{References}

1. Lloyd-Jones D, Adams RJ, Brown TM, et al. Heart disease and stroke statistics 2010 update: a report from the American Heart Association. Circulation. 2010;121:e46-e215.

2. Stehlik J, Edwards LB, Kucheryavaya AY, et al. The Registry of the International Society for Heart and Lung Transplantation: 29th official adult heart transplant report - 2012. J Heart Lung Transpl. 2012;31: 1052-1064.

3. Kirklin JK, Naftel DC, Pagani FD, et al. Sixth INTERMACS annual report: a 10,000 patients database. $J$ Heart Lung Transplant. 2014;33:555-564.

4. Gilotra NA, Russell SD. Patient selection for mechanical circulatory support. Heart Fail Rev. 2013;18:27-34.

5. Yancy CW, Jessup M, Bozkurt B, et al. 2013 ACCF/AHA Guideline for the Management of Heart Failure: a report of the American College of Cardiology Foundation/American Heart Association Task Force on Practice Guidelines. Circulation. 2013;128:e240-e327.

6. McMurray JJ, Adamopoulos S, Anker SD, et al. ESC guidelines for the diagnosis and treatment of acute and chronic heart failure 2012: The Task Force for the Diagnosis and Treatment of Acute and Chronic Heart Failure 2012 of the European Society of Cardiology. Eur J Heart Fail. 2012;14:803-869.

7. Peura JL, Colvin-Adams M, Francis GS, et al. American Heart Association Heart Failure and Transplantation Committee of the Council on Clinical Cardiology; Council on Cardiopulmonary, Critical Care, Perioperative and Resuscitation; Council on Cardiovascular Disease in the Young; Council on Cardiovascular Nursing; Council on Cardiovascular Radiology and Intervention, and Council on Cardiovascular Surgery and Anesthesia. Recommendations for the Use of Mechanical Circulatory Support: Device Strategies and Patient Selection A Scientific Statement from the American Heart Association. Circulation. 2012;126:2648-2667.

8. Fried LP, Ferrucci L, Darer J, et al. Untangling the concepts of disability, frailty, and comorbidity: implications for improved targeting and care. J Gerontol A Biol Sci Med Sci. 2004;59:255-263.

9. Flint KM, Matlock DD, Lindenfeld JA, Allen LA. Frailty and the selection of patients for destination therapy left ventricular assist device. Circ Heart Fail. 2012;5:286-293.

10. Teuteberg JJ, Ewald GA, Adamson RM, et al. Risk assessment for continuous flow left ventricular assist devices: does the destination therapy risk score work? An analysis of over 1,000 patients. J Am Coll Cardiol. 2012;60:44-51.

11. Yang JA, Kato TS, Shulman BP, et al. Liver dysfunction as a predictor of outcomes in patients with advanced heart failure requiring ventricular assist device support: use of the Model of End-stage Liver Disease (MELD) and MELD Excluding INR (MELD-XI) scoring system. $J$ Heart Lung Transplant. 2012;31:601-610.

12. Cowger J, Sundareswaran K, Rogers JG, et al. Predicting survival in patients receiving continuous flow left ventricular assist devices: the HeartMate II risk score. J Am Coll Cardiol. 2013;61:313-321.
13. Jorde UP, Kushwaha SS, Tatooles AJ, et al. Results of the destination therapy post-FDA-approval study with a continuous flow left ventricular assist device: a prospective study using the INTERMACS Registry (Interagency Registry for Mechanically Assisted Circulatory Support). J Am Coll Cardiol. 2014;63:1751-1757.

14. Atluri P, Goldstone AB, Fairman AS, et al. Predicting right ventricular failure in the modern, continuous flow left ventricular assist device era. Ann Thorac Surg. 2013;96:857-863.

15. Patlolla B, Beygui R, Haddad F. Right ventricular failure following left ventricle assist device implantation. Curr Opin Cardiol. 2013;28: 223-233.

16. Drakos SG, Janicki L, Horne BD, et al. Risk factors predictive of right ventricular failure after left ventricular assist device implantation. Am J Cardiol. 2010;105:1030-1035.

17. Pagani FD, Miller LW, Russell SD, et al. Extended mechanical circulatory support with a continuous-flow rotary left ventricular assist device. J Am Coll Cardiol. 2009;54:312-321.

18. Meineri M, Van Rensburg AE, Vegas A. Right ventricular failure after LVAD implantation: prevention and treatment. Best Pract Res Clin Anaesthesiol. 2012;26:217-229.

19. Grant AD, Smedira NG, Starling RC, et al. Independent and incremental role of quantitative right ventricular evaluation for the prediction of right ventricular failure after left ventricular assist device implantation. $J \mathrm{Am}$ Coll Cardiol. 2012;60:521-528.

20. Dandel M, Potapov E, Krabatsch T, et al. Load dependency of right ventricular performance is a major factor to be considered in decision making before ventricular assist device implantation. Circulation. 2013;128(11 Suppl 1):S14-S23.

21. Raina A, Seetha Rammohan HR, Gertz ZM, et al. Postoperative right ventricular failure after left ventricular assist device placement is predicted by preoperative echocardiographic structural, haemodynamic, and functional parameters. $J$ Card Fail. 2013;19:16-24.

22. Deswarte G, Kirsch M, Lesault PF, et al. Right ventricular reserve and outcome after continuous-flow left ventricular assist device implantation. J Heart Lung Transplant. 2010;29:1196-1198.

23. Morgan JA, Paone G, Nemeh HW et al. Impact of continuous-flow left ventricular assist device support on right ventricular function. $J$ Heart Lung Transplant. 2013;32(4):398-403.

24. Kormos RL, Teuteberg JJ, Pagani FD, et al. Right ventricular failure in patients with the HeartMate II continuous-flow left ventricular assist device: incidence, risk factors, and effect on outcomes. JThorac Cardiovasc Surg. 2010;139:1316-1324.

25. Horwich TB, Kalantar-Zadeh K, MacLellan RW, Fonarow GC. Albumin levels predict survival in patients with systolic heart failure. Am Heart J. 2008;155:883-889.

26. Melenovsky V, Kotrc M, Borlaug BA, et al. Relationships between right ventricular function, body composition, and prognosis in advanced heart failure. J Am Coll Cardiol. 2013;62:1660-1670.

27. Feldman D, Pamboukian SV, Teuteberg JJ, et al. 2013 ISHLT guidelines for mechanical circulatory support. J Heart Lung Transplant. 2013;32:157-187.

28. Bunte MC, Blackstone EH, Thuita L, et al. Major bleeding during HeartMate II Support. J Am Coll Cardiol. 2013;62:2188-2196.

29. Crow S, Chen D, Milano C, et al. Acquired von Willebrand syndrome in continuous-flow ventricular assist device recipients. Ann Thorac Surg. 2010;90:1263-1269.

30. Geisen U, Heilmann C, Beyersdorf F, et al. Non-surgical bleeding in patients with ventricular assist devices could be explained by acquired von Willebrand disease. Eur J Cardiothorac Surg. 2008;33: 679-684.

31. Boyle AJ, Jorde UP, Sun B, et al. Pre-operative risk factors of bleeding and stroke during left ventricular assist device support an analysis of more than 900 HeartMate II outpatients. J Am Coll Cardiol. 2014;63: $880-888$.

32. Aggarwal A, Pant R, Kumar S, et al. Incidence and management of gastrointestinal bleeding with continuous flow assist devices. Ann Thorac Surg. 2012;93:1534-1540. 
33. John R, Kamdar F, Liao K, et al. Improved survival and decreasing incidence of adverse events with the HeartMate II left ventricular assist device as bridge-to-transplant therapy. Ann Thorac Surg. 2008;86: 1227-1235.

34. Jennings DL, Jacob M, Chopra A, et al. Safety of anticoagulation reversal in patients supported with continuous-flow left-ventricular assist devices. ASAIO J. Epub 2014 March 20.

35. Interagency Registry for Mechanically Assisted Circulatory Support, National Heart Lung and Blood Institute. Adverse event definitions. Contract Award HHSN268201100025C. Available from: http:// www.uab.edu/medicine/intermacs/manual-of-operations/manual-ofoperations-dropdown3/appendix-a-adverse-event-definitions. Accessed December 12, 2013.

36. Cowger JA, Romano MA, Shah P, et al. Hemolysis: a harbinger of adverse outcome after left ventricular assist device implant. J Heart Lung Transplant. 2014;33:35-43.

37. Ravichandran AK, Parker J, Novak E, et al. Hemolyses in left ventricular assist device: a retrospective analysis of outcomes. J Heart Lung Transplant. 2014;33:44-50.

38. Kirklin JK, Naftel DC, Kormos RL, et al. Interagency Registry for Mechanically Assisted Circulatory Support (INTERMACS) analysis of pump thrombosis in the HeartMate II left ventricular assist device. $J$ Heart Lung Transplant. 2014;33:12-22.

39. Uriel N, Han J, Morrison KA, et al. Device thrombosis in HeartMate II continuous-flow left ventricular assist devices: a multifactorial phenomenon. J Heart Lung Transplant. 2014;33:51-59.

40. Uriel N, Morrison KA, Garan AR, et al. Development of a novel echocardiography ramp test for speed optimization and diagnosis of device thrombosis in continuous-flow left ventricular assist devices the Columbia Ramp Study. J Am Coll Cardiol. 2012;60:1764-1775.

41. Mishkin JD, Enriquez JR, Meyer DM, et al. Utilization of cardiac computed tomography angiography for the diagnosis of left ventricular assist device thrombosis. Circ Heart Fail. 2012;5:e27-e29.

42. Schlendorf K, Patel CB, Gehrig T, et al. Thrombolytic therapy for thrombosis of continuous flow ventricular assist devices. J Cardiac Fail. 2014;20:91-97.

43. Holman WL. Interagency Registry for Mechanically Assisted Circulatory Support (INTERMACS): what have we learned and what will we learn? Circulation. 2012;126:1401-1406.

44. Argiriou M, Kolokotron SM, Sakellaridis T, et al. Right heart failure post left ventricular assist device implantation. J Thorac Dis. 2014;6(S1): S52-S59.

45. Farrar DJ. Ventricular interactions during mechanical circulatory support. Semin Thorac Cardiovasc Surg. 1994;6:163-168.

46. Saito S, Sakaguchi T, Miyagawa S, et al. Recovery of right heart function with temporary right ventricular assist using a centrifugal pump in patients with severe biventricular failure. J Heart Lung Transplant. 2012;31:858-864.

47. John R, Mantz K, Eckman P, et al. Aortic valve pathophysiology during left ventricular assist device support. J Heart Lung Transplant. 2010; 29:1321-1329.

48. Pak SW, Uriel N, Takayama H, et al. Prevalence of de novo aortic insufficiency during long-term support with left ventricular assist devices. J Heart Lung Transplant. 2010;29:1172-1176.
49. Hatano M, Kinugawa K, Shiga T, et al. Less frequent opening of the aortic valve and a continuous flow pump are risk factors for postoperative onset of aortic insufficiency in patients with a left ventricular assist device. Circ J. 2011;75:1147-1155.

50. Cowger J, Pagani FD, Haft JW, et al. The development of aortic insufficiency in left ventricular assist device-supported patients. Circ Heart Fail. 2010;3:668-674.

51. Martina J, de Jonge N, Sukkel E, Lahpor J. Left ventricular assist devicerelated systolic aortic regurgitation. Circulation. 2011;124:487-488.

52. Cohn WE, Demirozu ZT, Frazier OH. Surgical closure of left ventricular outflow tract after left ventricular assist device implantation in patients with aortic valve pathology. J Heart Lung Transplant. 2011;30: 59-63.

53. Farrar DJ, Bourque K, Reichenbach SH, Muller P, Peri L. Innovation update. In: Kyo S, editor. Ventricular Assist Devices in Advanced-Stage Heart Failure. Japan, Tokyo: Springer; 2014. Available from: http:// issuu.com/chiasmatta/docs/4431544658. Accessed August 5, 2014.

54. Boyle A. Arrhythmias in patients with ventricular assist devices. Curr Opin Cardiol. 2012;27:13-18.

55. Maisel W, Stevenson L. Atrial fibrillation in heart failure: epidemiology, pathophysiology, and rationale for therapy. Am J Cardiol. 2003; 91 Suppl:2D-8D.

56. Oswald H, Schultz-Wildelau C, Gardiwal A, et al. Implantable defibrillator therapy for ventricular tachyarrhythmia in left ventricular assist device patients. Eur J Heart Fail. 2010;12:593-599.

57. Pedrotty DM, Rame JE, Marguilies KB. Management of ventricular arrhythmias in patients with ventricular assist devices. Curr Opin Cardiol. 2013;28:360-368.

58. Gregory SD, Timms D, Gaddum NR, et al. In vitro evaluation of a compliant inflow cannula reservoir to reduce suction events with extracorporeal rotary ventricular assist device support. Artif Organs. 2011;35: 765-772.

59. Klotz S, Foronjy RF, Dickstein ML, et al. Mechanical unloading during left ventricular assist device support increases left ventricular collagen crosslinking and myocardial stiffness. Circulation. 2005;112: 364-374.

60. Harding JD, Piacentino V 3rd, Gaughan JP, et al. Electrophysiological alterations after mechanical circulatory support in patients with advanced cardiac failure. Circulation. 2001;104:1241-1247.

61. Pereda D, Conte JV. Left ventricular assist device driveline infections. Cardiol Clin. 2011;29:515-527.

62. Hannan MM, Husain S, Mattner F, et al. Working formulation for the standardization of definitions of infections in patients using ventricular assist devices. J Heart Lung Transplant. 2011;30:375-384.

63. Pratt AK, Shah NS, Boyce SW. Left ventricular assist device management in the ICU. Crit Care Med. 2014;42:158-168.

64. Walker PC, DePestel DD, Miles NA, Malani PN. Surgical infection prophylaxis for left ventricular assist device implantation. J Card Surg. 2011;26:440-443.

65. Adzic A, Patel SR, Maybaum S. Impact of adverse events on ventricular assist device outcomes. Curr Heart Fail Rep. 2013;10:89-100.

Research Reports in Clinical Cardiology

\section{Publish your work in this journal}

Research Reports in Clinical Cardiology is an international, peerreviewed, open access journal publishing original research, reports, editorials, reviews and commentaries on all areas of cardiology in the clinic and laboratory. The manuscript management system is completely online and includes a very quick and fair peer-review system.

\section{Dovepress}

Visit http://www.dovepress.com/testimonials.php to read real quotes from published authors. 